\title{
Ischemia modified albumin in experimental ovarian torsion with and without controlled reperfusion
}

\author{
Cornelia Lazăr ${ }^{1}$, Marin Vozian², Valeriana Pantea ${ }^{3}$, Ana Mișina ${ }^{4}$, Olga Tagadiuc ${ }^{1}$ \\ 1. Biochemistry and Clinical Biochemistry Department, Nicolae Testemitanu State University \\ of Medicine and Pharmacy, Chişinău, Republic of Moldova
}

2. Surgery nr.1 "Nicolae Anestiadi" Department, Nicolae Testemitanu State University of Medicine and Pharmacy, Chişinău, Republic of Moldova

3. Laboratory of Biochemistry, Nicolae Testemitanu State University of Medicine and Pharmacy,

Chişinău, Republic of Moldova

4. Department of Surgical Gynecology, Mother and Child Institute, Chişinău, Republic of Moldova

\begin{abstract}
Purpose: Ovarian torsion, being a gynecological emergency, requires to be rapidly diagnosed and treated with minimal consequences on ovarian function after the removal of torsion. As ischemia modified albumin (IMA) is considered a good biomarker in diverse ischemic diseases, the aim of our study was to determine the effect of different ovarian torsion/detorsion models on serum and ovarian homogenates levels of IMA in an experimental study. Methods: IMA was measured in the serum and ovarian homogenates of 7 groups of female rats (10 animals in each group): 1 - control (no intervention); 2 - sham (only laparotomy); 3 - ischemia group: 3 hours ovarian torsion (OT); 4 - 3 hours OT (ischemia), 1 hour simple reperfusion; 5 - 3 hours ischemia, 1 hour controlled reperfusion that was assured during the first two minutes by opening and closing the clips on the ovarian pedicles in 10 seconds intervals, followed by simple reperfusion; 6 - 3 hours ischemia, 24 hours simple reperfusion; 7 - 3 hours OT, 24 hours controlled reperfusion. The results were analyzed by Welch's ANOVA and Spearman correlation. Results: Ischemia increases the IMA in both serum and ovarian homogenates compared to control and sham groups. The controlled reperfusion groups had a statistically significant lower IMA in serum compared to simple reperfusion groups. IMA was found to be higher in the ovarian homogenates of simple reperfusion compared to controlled reperfusion groups. Conclusion: Our results suggest that controlled reperfusion prevent the processes that increase the IMA in ovarian torsion.
\end{abstract}

Keywords: ovarian torsion, ischemia modified albumin

Received: $8^{\text {th }}$ November 2018; Accepted: 28 th December 2018; Published: $31^{\text {st }}$ December 2018

*Corresponding author: Cornelia Lazăr, Nicolae Testemitanu State University of Medicine and Pharmacy Chișinău, Republic of Moldova. E-mail: cornelia.lazar@usmf.md 


\section{Introduction}

Being a gynecological emergency $(1,2)$, the torsion of the ovary is a partial or complete rotation of the ovary, the fallopian tube or both of them around their vascular axis (3). Of the total torsional cases, $70-80 \%$ occur in women of reproductive age (4). The incidence is $4.9 / 100000$ in women under the age of $20(1,5,6,7,8)$, but it may affect women at any age $(9,10)$.

The first choice in the treatment is to detorsion the torsioned anexa, but it is well known that reperfusion can increase the initial ischemic injuries because it determines reactive oxygen species (ROS) production (11) leading to a high level of oxidative stress and reperfusion lesions. Reperfusion injury describes the events associated with restoration of blood flow that did not occur during the ischemia period and can be prevented by an intervention given only at the moment of reperfusion (12).

IMA is a biomarker of oxidative stress in hypoxic conditions (13). High levels of IMA are detected in diseases associated with ischemic processes $(14,15)$ due to modification of the serum albumin N-terminal end $(16,17,18)$. Increase of IMA in ovarian torsion was shown in different studies $(2,9,10,19)$. In the acute ischemia IMA is a product of ROS interaction with the protein albumin (20).

The N-terminal end of albumin has a sequence aspartate-alanine-histidine-lysine that is responsible for $\mathrm{Co}^{2+}$ binding (17). Hydroxyl radical interaction with the $\mathrm{N}$-terminal end of albumin determines the changes that release the $\mathrm{Co}^{2+}(21)$. ROS derived from a torsioned ovary are involved in the reduction of albumin capacity to bind transition metals. This leads to the formation of a new variant of albumin known as IMA (10). It is a nonspecific marker for ischemia because ROS can be formed in every kind of ischemia (9). ROS production increases in the reperfusion period, thus we suppose that the technique of reperfusion is important to prevent the growth of ischemic lesions.

There are no studies in the literature that examine the IMA in serum and ovarian homogenates in different types of reperfusion for ovarian torsion. The relationship between controlled reperfusion and IMA levels in the ovarian torsion was not studied before.

The aim of our research was to investigate the effect of different ovarian torsion/detorsion models on serum and ovarian homogenates IMA levels and to appreciate the effectiveness of the controlled reperfusion method in reducing the reperfusion injuries.

\section{Material and methods}

Seventy healthy female rats (Rattus albicans), weighing 180-265 grams, were taken from the Nicolae Testemitanu State University of Medicine and Pharmacy vivarium. All manipulations were made in the Laboratory of Biochemistry of Nicolae Testemitanu State University of Medicine and Pharmacy. The animals were used and maintained in accordance with the Animal Welfare Act. The Research Ethics Committee of Nicolae Testemitanu State University of Medicine and Pharmacy, Republic of Moldova, approved our study protocol (08.02.2016).

The rats were randomly divided into seven experimental groups $(n=10)$ : group 1: control (no intervention); group 2: sham (only laparotomy); group 3: ischemia (ovarian torsion for 3 hours); group 4: ischemia (ovarian torsion for 3 hours) and 1 hour simple reperfusion; group 5: ischemia (ovarian torsion for 3 hours) and 1 hour controlled reperfusion; group 6: ischemia (ovarian torsion for 3 hours) and 24 hours simple reperfusion; group 7: ischemia (ovarian torsion for 3 hours) and 24 hours controlled reperfusion.

Surgical procedures were done under sterile conditions and anesthesia. The animals were anesthetized with $10 \%$ ketamine hydrochloride sol., 
50 mg/kg (Calypsol®, Gedeon Richter, Hungary) administered intraperitoneally. The time was considered appropriate for surgical intervention when the animals remained motionless in a supine position. The abdominal skin was shaved and disinfected with Iodine povidone $100 \mathrm{mg} / \mathrm{ml}$ (Betadine $\AA$, Egis Pharmaceuticals PLC, Hungary). The animals breathed spontaneously and were positioned in dorsal decubitus. The intervention time was approximately 15 minutes. A 2-2.5 cm laparatomy was performed - a vertical incision in the lower abdomen. The rats from group 1 (control) were anesthetized, the ovaries were removed and blood collected from the abdominal aorta, after which the animals were euthanized. The rats from group 2 (sham) were anesthetized, laparatomy was performed and after 15 minutes the abdominal wall was closed by applying $3 / 0$ silk sutures. After 3 hours the animals were anesthetized, re-laparatomy was performed, the ovaries were removed and blood from the abdominal aorta colected, after which the animals were euthanized.

In groups 3, 4, 5, 6 and 7 the ovarian torsion was induced by twisting the annexes three times in clockwise direction. After twisting, the annexes were fixed to the abdominal wall by applying 3/0 silk suture and then the abdominal wall was closed by applying 3/0 silk sutures. After 3 hours of torsion the rats were anesthetized, re-laparatomy was performed and according to the group they belong, the ovaries were removed and blood collected from the abdominal aorta for group 3, or simple detorsion (simple reperfusion) was performed for 1 hour for group 4 , and 24 hours for group 6, or controlled detorsion (controlled reperfusion) for 1 hour for group 5, and 24 hours for group 7 . The controlled reperfusion was performed by opening and closing the clips that were applied on the ovarian annexes (on/off) in 10-seconds intervals for the first 2 minutes of reperfusion. The abdominal wall was closed by applying 3/0 silk sutures. At the end of the reperfusion period the animals were anesthetized, re-laparatomy was performed with the removal of the ovaries and blood samples colection from the abdominal aorta, after which the animals were euthanized.

The blood samples were placed into tubes allowing to clot for 30 minutes, then centrifuged 10 minutes at $3000 \mathrm{rpm}$. Serum was stored at $-40^{\circ}$ $\mathrm{C}$ until analysis.

The ovarian tissue $(0,2 \mathrm{~g})$ was homogenized in ice with $2 \mathrm{ml}$ of phosphate buffer $0.1 \mathrm{M}$ $(\mathrm{pH}=7.4)$, centrifuged at $4^{\circ} \mathrm{C}, 3000 \mathrm{rpm}$ for 15 minutes. The supernatant stored at $-40^{\circ} \mathrm{C}$ until analysis.

The IMA levels were determined in blood serum and ovarian homogenates samples according to the modified cobalt-binding assay described by Gudumac V. and Tagadiuc O. (22).

The obtained data were analysed using SPSS 20.0 software. Descriptive statistical methods (mean, standard error of the mean) were used. Normality tests were done using the KolmogorovSmirnov and Shapiro-Wilk tests. The homogeneity of variance was tested using Levene's test. The comparisons between groups were performed using Welch's ANOVA with Games-Howell post hoc test. Correlation analysis was done using Spearman correlation test. A p $<0.05$ value was considered statistically significant.

\section{Results}

There is a statistically significant increase of IMA levels in serum in the sham group compared to control group, from $402.370 \pm 2.732 \mu \mathrm{mol} / \mathrm{L}$ to $418.472 \pm 1.854 \mu \mathrm{mol} / \mathrm{L}(\mathrm{p}=0.003)$ (figure 1$)$. Ischemia (group 3 ) determined IMA increase up to $478.359 \pm 5.218 \mu \mathrm{mol} / \mathrm{L}$ compared to control and sham groups $(\mathrm{p}<0.001)$. Statistically significant high levels of IMA were registered at 1 hour $(490.024 \pm 3.376 \mu \mathrm{mol} / \mathrm{L})$ and 24 hours $(483.370 \pm 1.550 \mu \mathrm{mol} / \mathrm{L})$ of simple reperfusion compared to 1 hour $(452.564 \pm 3.096 \mu \mathrm{mol} / \mathrm{L}$, 


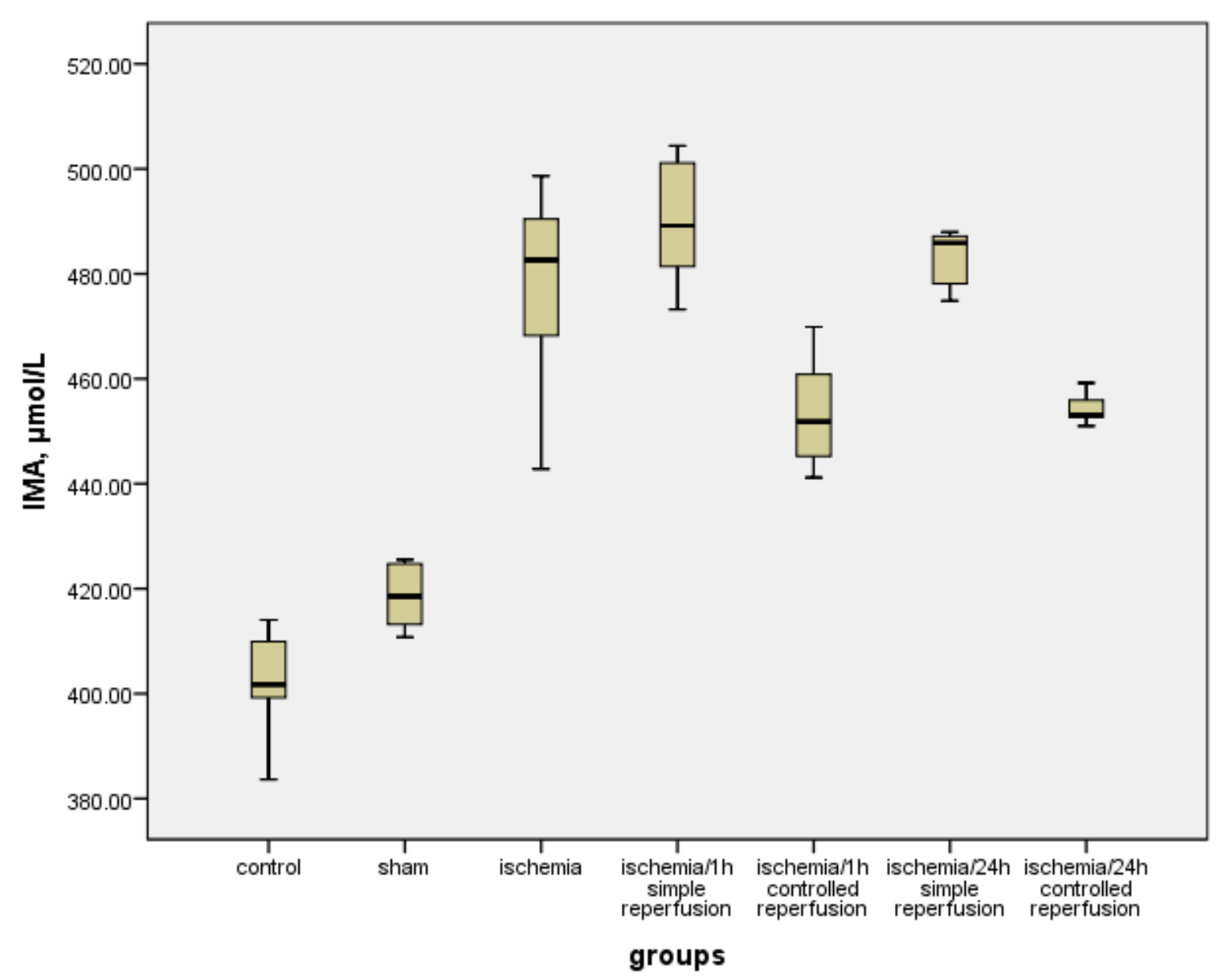

Fig1. IMA values in serum

$\mathrm{p}<0.001)$ and 24 hours $(454.207 \pm 0.878 \mu \mathrm{mol} / \mathrm{L}$, $\mathrm{p}<0.001)$ of controlled reperfusion.

In the ovarian homogenates (figure 2) the IMA values were $14.233 \pm 0.458 \mu \mathrm{mol} / \mathrm{g}$.prot. in the control group, $14.251 \pm 0.733 \mu \mathrm{mol} / \mathrm{g}$.prot. in the sham group, $15.274 \pm 0.284 \mu \mathrm{mol} / \mathrm{g}$.prot. in the ovarian torsion (ischemia) group, $17.384 \pm 1.018$ $\mu \mathrm{mol} / \mathrm{g}$.prot. in the ovarian torsion/1 hour simple reperfusion group, $16.423 \pm 1.618 \mu \mathrm{mol} / \mathrm{g}$. prot. in the ovarian torsion/ 1 hour controlled reperfusion group, $16.855 \pm 1.211 \mu \mathrm{mol} /$ g.prot. in the ovarian torsion/24 hours simple reperfusion group, $16.106 \pm 1.154 \mu \mathrm{mol} / \mathrm{g}$.prot. in the ovarian torsion/24 hours controlled reperfusion group. There were no statistically significant differences between groups ( $\mathrm{p}>0.05$ ).

No correlations were found between the serum IMA levels and the one registered in ovarian homogenates.

\section{Discussion}

Diseases characterized by acute ischemia are considered medical emergencies and the main treatment tactic involves the restoration of the tissue blood flow to prevent the necrosis. However, after an ischemic period, reperfusion can increase the initial ischemic injuries due to ROS 


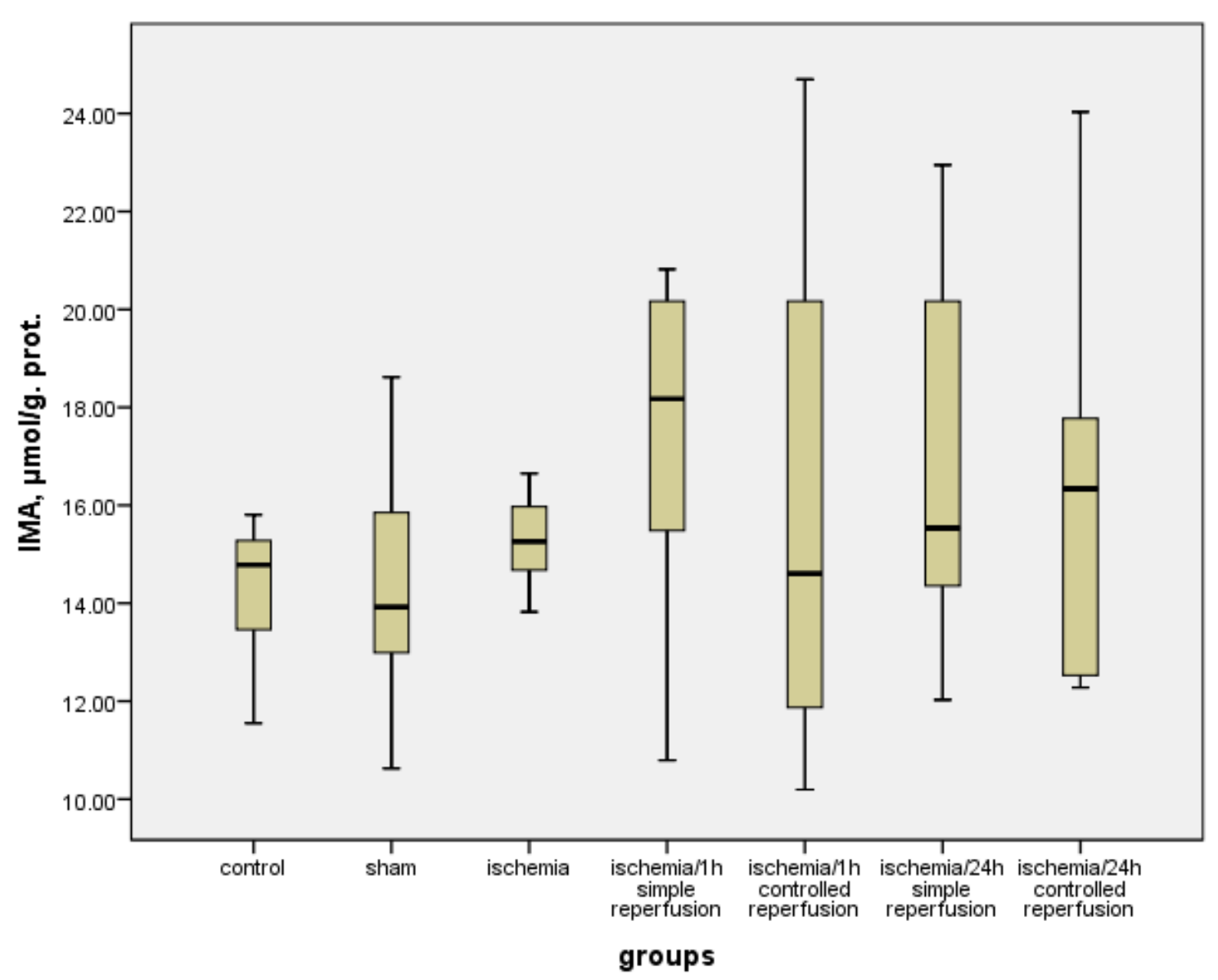

Fig2. IMA values in ovarian homogenates.

production. ROS can change different molecules, one of them being the protein albumin found in the blood. The changes in the structure of this protein lead to the formation of IMA. So, the new protein that originates from albumin is produced during the ischemic period and in the reperfusion phase it is possible to increase its amounts because of an intensive oxidative stress. It is well known that the amounts of IMA begin to rise within minutes following an ischemic process $(14,15)$.

The concept of controlled reperfusion was applied by different researchers in different pathologies including the torsion of the ovaries. In the experimental study done by Ingec M. et al. where a similar method of reperfusion for ovarian torsion was used as was for our study, the damage markers related to oxidative stress (malondialdehyde and 8-hydroxyguanine) were found to be lower in the controlled reperfusion group (23). The Ozkisacik S. et al. research with a different study design for ischemia/reperfusion injury in ovarian torsion concluded that gradual detorsion can reduce the reperfusion injury (24). As in our study, the Aran T. et al. experiment has shown that ovarian torsion increases significantly the serum IMA levels compared to the control group (9). Another research conducted by Yildirim A. et al. has found higher serum IMA in the ovarian torsion group compared to control and 
also a strong correlation with the histopathologic changes (19).

Contrary to our findings and the studies done by Aran T. et al. and Yildirim A. et al., the research of Karatas Gurgun A. et al. did not find significant increase in the IMA levels in the torsion groups suggesting that IMA may gain more significance with increasing duration of ischemia (2).

In our research, IMA levels registered in homogenates were not significantly different, but there is a tendency for them to increase after the torsion of the ovary with approximately $7.3 \%$ compared to the control group. The amount of IMA registered at 1 hour of simple reperfusion after detorsion was by $22.1 \%$ higher than those recorded in the control group. This fact confirms once again that ischemia activates the processes responsible for albumin modification, decreasing its capacity to bind $\mathrm{Co}^{2+}$. Simple reperfusion increasing the oxidative stress stimulates the IMA formation, confirming the protein changes after restoration of blood flow.

As was shown in other studies with ischemia and reperfusion, ischemia is dangerous, but reperfusion can be worse (25). The same thing is confirmed by our results. The simple reperfusion groups had higher IMA levels compared to ischemia group in both serum and ovarian homogenates. However, the controlled reperfusion groups had a statistically significant low IMA in serum compared to simple reperfusion groups. IMA was found to be higher in the ovarian homogenates of simple reperfusion compared to controlled reperfusion groups also. Therefore, the type of reperfusion can influence the IMA production.

The lack of correlation between serum IMA and homogenate IMA suggests the need for careful interpretation of the increase of this biomarker in the blood, and that it should always be viewed in a clinical context.

There is one clinical study investigating the amounts of IMA in patients with pelvic pain, in- dicating that elevated serum concentrations and clinical suspicion of ovarian torsion is a situation when doctors can decide to do a laparoscopy to exclude the torsion (10).

\section{Conclusion}

Ischemia and reperfusion can increase protein changes and the IMA level in experimental ovarian torsion. Controlled reperfusion reduces the protein damage, registered by a decreased level of IMA in blood serum samples of controlled reperfusion groups. Decreased levels of IMA in controlled reperfusion groups indicate that this type of reperfusion is able to protect the organ from ROS production and to minimize the reperfusion injury in experimental ovarian torsion. The IMA should be examined in clinical context.

\section{Research funding}

Doctoral grant offered by Ministry of Education, Culture and Research of Republic of Moldova.

\section{Author's contribution}

C.L. - study design, surgical procedures, data analysis and interpretation, wrote the manuscript with support by O.T., approved the final manuscript

M.V. - surgical procedures, participation in the collection of biological samples for biochemical investigations, read and approved the manuscript V.P. - biochemical analysis, final approval of the manuscript

A.M. - surgical procedures, revised the manuscript and approved the final version

O.T. - study design, biochemical analysis, data analysis and interpretation, critical revision, final approval of the version to be published

\section{Conflict of interest}

No conflicts of interest. 


\section{Abbreviations \\ IMA - Ischemia modified albumin \\ OT - Ovarian torsion \\ ROS - Reactive oxygen species}

\section{References}

1. McCloskey K, Grover S, VuillerminBabl FE. Ovarian torsion among girls presenting with abdominal pain: a retrospective cohort study. Emerg Med J. 2013; 30(1):e11. DOI: 10.1136/emermed-2011-200200

2. Karatas Gurgun A, Kaban I, Aka N, Mentese A, Aker F, Uras AR. The Role of ischemia Modified Albumin and D-dimer as Early or Late Biochemical Markers in Ovarian Torsion. J Obstet Gynaecol Res. 2017; 43(5):895-901. DOI: 10.1111/jog.13281

3. Tuncer AA, Bozkurt MF, Koken T, Dogan N, Pektaş MK, Baskin Embleton D. The Protective Effects of Alpha-Lipoic Acid and Coenzyme Q10 Combination on Ovarian Ischemia-Reperfusion Injury: An Experimental Study. Adv Med. 2016; 2016:3415046. DOI: 10.1155/2016/3415046

4. Zucchini S, Marra E. Diagnosis of emergencies/urgencies in gynecology and during the first trimester of pregnancy. J Ultrasound. 2014; 17(1):41-6. DOI: 10.1007/s40477-013-0059-0

5. Childress KJ, Dietrich JE. Pediatric ovarian torsion. Surg Clin North Am. 2017; 97(1):209-21. DOI: 10.1016/j.suc.2016.08.008

6. Spinelli C, Piscioneri J, Strambi S. Adnexal torsion in adolescents: update and review of the literature. Curr Opin Obstet Gynecol. 2015; 27(5):320-5. DOI: 10.1097/GCO.0000000000000197

7. Karaman E, Beger B, Çetin O, Melek M, Karaman Y. Ovarian Torsion in the Normal Ovary: A Diagnostic Challenge in Postmenarchal Adolescent Girls in the Emergency Department. Med Sci Monit. 2017; 23:1312-6. DOI: 10.12659/MSM.902099

8. BolliSchädelin S, Holland-Cunz S, ZimmermannOvarian torsion in children: Development of a predictive score. Medicine. 2017; 96(43):e8299. DOI: 10.1097/ MD.0000000000008299

9. Aran T, Guven S, Unsal MA, Alver A, Mentese A, Yulug E. Serum ischemia-modified albumin as a novel marker of ovarian torsion: an experimental study. Eur J Obstet Gynecol Reprod Biol. 2010; 150(1):72-5. DOI: 10.1016/j.ejogrb.2010.01.006
10. Guven S, Kart C, Guven ESG, Cetin EC, Menteşe A. Is the measurement of serum ischemia-modified albumin the best test to diagnose ovarian torsion? Gynecol Obstet Invest. 2015; 79(4):269-75. DOI: 10.1159/000367787

11. Laganà AS, Sofo V, Salmeri FM, Palmara VI, Triolo O, Terzić MM, et al. Oxidative stress during ovarian torsion in pediatric and adolescent patients: changing the perspective of the disease. Int J Fertil Steril. 2016; 9(4):416-23.

12. Kalogeris T, Baines CP, Krenz M, Korthuis RJ. Cell Biology of Ischemia/Reperfusion Injury. Int Rev Cell Mol Biol. 2012; 298:229-317. DOI: 10.1016/B978-012-394309-5.00006-7

13. Żurawska-Płaksej E, Grzebyk E, Marciniak D, Szymańska-Chabowska A, Piwowar A. Oxidatively modified forms of albumin in patients with risk factors of metabolic syndrome. J Endocrinol Invest. 2014; 37(9):819-27. DOI: 10.1007/s40618-014-0111-8

14. Mertoglu C, Gunay M, Gurel A, Gungor M, Gul V. Ischemic modified albumin increases in acute kidney injury. Rev Romana Med Lab. 2018; 26(1):37-43. DOI: 10.2478/rrlm-2018-0002

15. Shen XL, Lin CJ, Han LL, Lin L, Pan L,XD. Assessment of ischemia-modified albumin levels for emergency room diagnosis of acute coronary syndrome. Int J Cardiol. 2011; 149(3):296-8. DOI: 10.1016/j.ijcard.2010.01.013

16. Gaze DC. Ischemia Modified Albumin: a novel biomarker for the detection of cardiac ischemia. Drug Metab Pharmacokinet. 2009; 24(4):333-41. DOI: 10.2133/ dmpk.24.333

17. Bar-Or D, Curtis G, Rao N, Bampos N, Lau E. Characterization of the $\mathrm{Co}(2+)$ and $\mathrm{Ni}(2 \square)$ binding amino-acid residues of the N-terminus of human albumin. An insight into the mechanism of a new assay for myocardial ischemia. Eur J Biochem. 2001; 268(1):42-7. DOI: 10.1046/j.1432-1327.2001.01846.x

18. Liyan C, Jie Z, Yonghua W, Xiaozhou H. Assay of ischemia-modified albumin and c-reactive protein for early diagnosis of acute coronary syndromes. J Clin Lab Anal. 2008; 22(1):45-9. DOI: 10.1002/jcla.20223

19. Yildirim A, Yildirim S, Topaloglu N, Tekin M, Kucuk A, Erdem H, et al. Correlation of ischemia-modified albumin levels and histopathologic findings in experimental ovarian torsion. Turk J Emerg Med. 2016; 16(1):8-11. DOI: 10.1016/j.tjem.2016.02.004

20. Lippi G, Montagnana M, Guidi GC. Albumin cobalt 
binding and ischemia modified albumin generation: an endogenous response to ischemia? Int J Cardiol. 2006; 108(3):410-11. DOI: 10.1016/j.ijcard.2005.03.040

21. Marx G, Chevion M. Site-specific modification of albumin by free radicals. Reaction with copper (II) and ascorbate. Biochem J. 1986; 236(2):397-400. DOI: 10.1042/bj2360397

22. Gudumac V, Tagadiuc O. Metodă de determinare a capacităţii albuminei ischemic modificate de legare a cobaltului. Brevet de invenție nr. MD 4054 din 11.06.2009 http://www.db.agepi.md/Inventions/details/a\%202009\%200116/Des a\%202009\%200116.

23. Ingec M, Isaoglu U, Yilmaz M, Calik M, Polat B, Alp
$\mathrm{HH}$, et al. Prevention of ischemia-reperfusion injury in rat ovarian tissue with the on-off method. J Physiol Pharmacol. 2011; 62(5):575-82.

24. Ozkisacik S, Yazici M, Gursoy H, Culhaci N. Does gradual detorsion protect the ovary against ischemia-reperfusion injury in rats? Pediatr Surg Int. 2014; 30(4):437-40. DOI: 10.1007/s00383-014-3480-3

25. Gencer M, Karaca T, Güngör AN, Hacıvelioğlu SÖ, Demirtaş S, Turkon H, et al. The protective effect of quercetin on IMA levels and apoptosis in experimental ovarian ischemia-reperfusion injury. Eur J Obstet Gynecol Reprod Biol. 2014; 177:135-40. DOI: 10.1016/j. ejogrb.2014.03.036 